\title{
Modulation of Cosmic Rays at Different Cutoff Rigidity
}

\author{
Rekha Agarwal ${ }^{1}$ and Rajesh K. Mishra ${ }^{2}$ \\ ${ }^{1}$ Department of Physics, Govt. Model Science College (Autonomous), Jabalpur (M.P.) 482 001, India \\ ${ }^{2}$ Computer and IT Section, Tropical Forest Research Institute, \\ P.O. RFRC, Mandla Road, Jabalpur (M.P.) 482 021, India
}

Received on 13 September, 2007

\begin{abstract}
The present work deals with the study of first three harmonics of cosmic ray intensity on geo-magnetically quiet days over the period 1981-1993 for Deep River and Inuvik neutron monitoring stations having mid and low cutoff rigidity. The amplitude of first harmonic remains high for Deep River having mid cutoff rigidity as compared to Inuvik neutron monitor having low cutoff rigidity on quiet days. The diurnal amplitude significantly decreases and phase shifts towards an earlier time during solar activity minimum years at both Deep River and Inuvik. The amplitude of second harmonic significantly enhanced during solar activity minimum as well as maximum at Deep River and remains low during solar activity maximum at Inuvik, whereas the phase shifts towards an earlier time during solar maximum for both the stations. The amplitude of the third harmonic significantly enhanced during solar activity minimum at Deep River and on solar activity minimum at Inuvik, whereas the phase does not show any significant characteristics and fluctuates quite frequently. The amplitude of semi/tri-diurnal anisotropy has a good positive correlation, while the others (i.e. amplitude and phase) have very weak correlation with solar wind velocity on quiet days at Deep River station during 1981-1993. The solar wind velocity significantly remains in the range 350 to $425 \mathrm{~km} / \mathrm{s}$ i.e. being nearly average on quiet days. The amplitude and direction of the anisotropy on quiet days are weakly dependent on high-speed solar wind streams for two neutron monitoring station of mid and low cutoff rigidity threshold. The amplitude as well as direction of second harmonic has a good anti-correlation with IMF Bz and the product $\mathrm{V} x \mathrm{Bz}$ on quiet days at Deep River station. However, the direction of second and third harmonic has a good anti-correlation with IMF Bz and the product $\mathrm{V} \times \mathrm{Bz}$ on quiet days at Inuvik station.
\end{abstract}

Keywords: Cosmic ray; Quiet days; Diurnal; Semi-diurnal anisotropy; Solar wind; Interplanetary magnetic field and geomagnetic activity

\section{INTRODUCTION}

Cosmic ray anisotropic variations and their characteristics are studied through the diurnal and semi-diurnal components mainly and the level of the isotropic intensity collectively provides fingerprint for identifying the modulating process and the electromagnetic state of interplanetary space in the neighborhood of the Earth. Many workers have attempted to derive relationship between the mean daily variation and the level of solar and geomagnetic activity (Venkatesan and Badruddin, 1990). Yearly average values of the first harmonic of solar daily variation experience strong changes from year to year and with the cycle of solar activity. Amplitude and phase of diurnal anisotropy is changed with the solar activity cycles (Ahluwalia and Fikani, 1997; El-Borie et al., 1995; Sikripin and Mamrukova, 1993). Lockwood and Webber (1984) found a close relationship between the magnitude and frequency of Forbush decreases and the eleven-year cosmic ray variation. They concluded that the effect of Forbush and other transient decreases is a dominant factor in the long-term intensity modulation. Forbush (1979) showed that annual means of the CR diurnal anisotropy resulted from the addition of two distinct components. One, $\mathrm{W}$ has its maximum in the asymptotic direction of $128^{\circ} \mathrm{E}$ of the Sun and is well approximated by a wave $\mathrm{W}$ with a period of two solar cycles and the other component $\mathrm{V}$ has its maximum in the asymptotic direction $90^{\circ}$ E of the Sun. Ahluwalia (1988) has reported that diurnal anisotropy is unidirectional during 1957-70 with direction along $1800 \mathrm{Hr}$ LT (East-West) and during 1971-79 it consists of two components; one is in the East-West direction and the other is the radial component with direction along $1200 \mathrm{Hr}$ LT. Sabbah (1998) characterized the diurnal anisotropy by two components. Only one anisotropy is dominant during each magnetic state of the solar cycle. The direction of the dominant anisotropy vector points towards the $1800 \mathrm{Hr}$ LT direction during the negative state of the solar cycle and toward earlier hours during the positive state. Ballif et al. (1969) correlated $\mathrm{Kp}$ and Ap with the mean fluctuations in amplitude of IMF, which in turn is related to diffusive component of convection-diffusion theory. Ap is also found to related with solar wind velocity, which is related to the convective component of convection-diffusion theory. Agrawal (1983) and Bieber and Evenson (1997) have preferred to investigate the daily variation in cosmic ray intensity on long/short term basis performing the analysis for all days in a year; whereas, Kumar et al. $(1981,1993)$ have studied long/short term daily variation on geomagnetically 60 quiet days (QD). Jadhav et al. (1983) and Kumar et al. (1999) studied daily variation during days of low and high amplitude anisotropic wave trains. Sabbah (1999) calculated the diurnal variation for days with high, intermediate and low interplanetary magnetic field (IMF) magnitude.

Special type of consecutive days having abnormally high or low amplitudes in daily variation of cosmic rays have been reported several times earlier with explanation of sources and sinks in anti-garden-hose and garden-hose directions (Jadhav et al., 1983; Rao et al., 1972; Tiwari, 1994). The existence of high and low amplitude anisotropic wave trains have been revealed through the long-term study of cosmic ray intensity. 
Periods of unusually large amplitude often occur in trains of several days. The average characteristics of cosmic-ray diurnal anisotropy are adequately explained by the co-rotational concept (Parker, 1964; Axford, 1965a, b). This concept supports the mean diurnal amplitude in space of $0.4 \%$ along the $1800 \mathrm{Hr}$ direction using the worldwide neutron monitor data. However, the observed day-to-day variation both in amplitude and time of maximum, and the abnormally large amplitudes or abnormally low amplitudes of consecutive days, cannot be explained in co-rotational terms. Moreover, the maximum intensity of diurnal anisotropy has not appeared in the direction of $1800 \mathrm{Hr}$, which is the nominal co-rotational phase (McCraken and Rao, 1965; Rao, 1972).

The average daily variation of cosmic ray intensity generally consists of diurnal variation, semi-diurnal variation and tri-diurnal variation. The amplitude of the diurnal variation at a high / middle latitude station has been found to be of the order of 0.3 to $0.4 \%$, whereas the amplitudes of two higher harmonics is of the order of $0.02 \%$ and $0.08 \%$ respectively (Pomerantz et al., 1960). The average characteristics have also been found to vary with solar cycle, where the variation is much larger at higher energies.

A number of investigators have reported the short-term characteristics of the daily variation, where they have selected continually occurring days of high and low amplitudes of diurnal variation (Tiwari, 1994; Agrawal et al., 1974, Tiwari, 1995). These results have pointed out significant departures in the time of maximum as well as their association with higher harmonics.

Many workers (Rao et al., 1972; Kane, 1974; Owens and Kash, 1976) used a new concept for the interpretation of the diurnal variation. McCraken et al. (1968) first suggested the extension of this new concept from the solar cosmic events to the observed diurnal variation and theoretical formulation has provided by Forman and Gleeson (1975). Several workers have attempted to find the possible origin of the 'large amplitude wave trains' of cosmic ray neutron intensity to develop a suitable realistic theoretical model, which can explain the diurnal anisotropy in individual days.

Hashim and Thambyahpillai (1969) and Rao et al. (1972) have shown that the enhanced diurnal variation of large amplitude events exhibits a maximum intensity in space around the anti-garden-hose direction $(2100 \mathrm{Hr})$ and a minimum intensity in space around the garden-hose direction $(0900 \mathrm{Hr})$. Kane (1970) and Bussoletti (1973) have noticed that quite often an enhanced intensity is presented along the corotational direction and it is not correlated with the garden-hose direction.

The diurnal anisotropy is well understood in terms of a convective-diffusive mechanism (Forman and L.J. Gleeson, 1975). Mavromichalaki (1979) has observed that the enhanced diurnal variation was caused by a source around 1600 $\mathrm{Hr}$ or by a sink at about $0400 \mathrm{Hr}$. It was pointed out that this diurnal variation by the superposition of convection and fieldaligned diffusion due to an enhanced density gradient of $\approx 8 \%$ $\mathrm{AU}^{-1}$.

\section{ANALYSIS OF DATA}

The amplitude and phase of the harmonics of the daily variation in cosmic ray intensity are derived by Fourier Analysis (Chapmen and Bartels, 1940), by noting the hourly counting rate of the observed cosmic ray intensity over a period of twenty-four hours.

The Fourier analysis yields reliable measures of the amplitude and phase on a day-to-day basis, provided the time series is reasonably stationary. However, this method cannot estimate the amplitude of the ambient anisotropy, which, for small amplitudes, contributes to large uncertainties in the Fourier coefficients.

\section{HARMONIC ANALYSIS}

Time dependent harmonic function $F(t)$ with 24 equidistant points in the interval from $t=0$ to $t=2 \pi$ can be expressed in terms of Fourier series

$$
\begin{gathered}
F(t)=a_{0}+\sum_{N=1}^{24}\left(a_{n} \cos (n t)+b_{n} \sin (n t)\right) \\
\left.F(t)=a_{0}+\sum_{N=1}^{24} r_{n} \cos (n t)-\phi_{n}\right)
\end{gathered}
$$

Where $a_{0}$ is the mean value of $F(t)$ for the time interval from $t=0$ to $2 \pi$ and $a_{n}, b_{n}$ are the coefficients of $n^{t h}$ harmonics, which can be expressed as follows:

$$
\begin{gathered}
a_{0}=\frac{1}{12} \sum_{i=1}^{24} n \\
a_{n}=\frac{1}{12} \sum_{i=1}^{24} n \cos n t \\
b_{n}=\frac{1}{12} \sum_{i=1}^{24} n \sin n t
\end{gathered}
$$

The amplitude $r_{n}$ and phase $\phi_{n}$ of the $n^{\text {th }}$ harmonic are expressed as

$$
r_{n}=\left(a_{n}^{2}+b_{n}^{2}\right)^{1 / 2}
$$

and

$$
\phi_{n}=\tan ^{-1}\left[\frac{a_{n}}{b_{n}}\right]
$$

The daily variation of the cosmic ray intensity can be adequately represented by the superposition of first, second, third and fourth harmonics as follows:

$$
\begin{gathered}
F(t)=a_{1} \cos t+b_{1} \sin t+a_{2} \cos 2 t+b_{2} \sin 2 t+ \\
a_{3} \cos 3 t+b_{3} \sin 3 t+a_{4} \cos 4 t+b_{4} \cos 4 t
\end{gathered}
$$




\section{TREND CORRECTION}

The daily variation in cosmic ray intensity is not strictly periodic. Thus, if the number to be analysed represents bihourly (or hourly) means of cosmic ray intensity, the mean for hour $t_{0}\left(0^{\text {th }}\right.$ hour) will not, in general be the same as the mean for hour $t_{24}$ (or $24^{\text {th }}$ hour) this difference on account of secular changes, is allowed for in practice by applying a correction known as trend correction, to each of the terms.

If $y_{0}$ is the value of the ordinate at $x=0\left(0^{\text {th }}\right.$ hour $)$ and $y_{12}$ is the value of the ordinate at $x=2 \pi\left(24^{\text {th }}\right.$ hour) then the trend corrected value for any hour is given by the equation

$$
\bar{y}_{k}=y_{k} \frac{\left( \pm \delta_{y} \times k\right)}{12}
$$

Where $k=0,1,2,3, . .12$

$y_{k}=$ uncorrected value

$\pm \delta_{y}=$ secular changes i.e. $\pm \boldsymbol{\delta}_{y}=y_{12}-y_{0}$

\section{MODE OF ANALYSIS}

The pressure corrected data (data from http://spidr.ngdc.noaa.gov/ NeutronMonitor) of Deep River (Vertical cutoff rigidity $=1.02 \mathrm{GV}$, Geog. Latitude = $46.1^{\circ} \mathrm{N}$, Geog. Longitude $=282.5^{\circ} \mathrm{E}$ ) and Inuvik (Vertical cutoff rigidity $=0.18 \mathrm{GV}, \mathrm{Geog}$. Latitude $=68.35^{\circ} \mathrm{N}$, Geog. Longitude $=226.27^{\circ} \mathrm{E}$ ) Neutron Monitor $(\mathrm{NM})$ station has been subjected to Fourier analysis for the period 1981-93 after applying the trend correction. While performing the analysis of the data all those days are discarded having more than three continuous hourly data missing.

\section{CRITERIA FOR SELECTION OF 60 QUIET DAYS}

Days on which the transient magnetic variations are regular and smooth are said to be magnetically quiet or calm or $\mathrm{Q}$ days. These are the days with low values of Ap and Kp. According to solar geophysical data (SGD) five quietest days in a month thus, $60 \mathrm{Q}$ days in a year are selected. These days are called the International quiet-quiet-days or QQ days. Kumar et al. (Kumar et al., 1993, 1998) have studied long/short term daily variation on geomagnetically 60 QD. The 60 QD are better suited for long/short term studies of daily variation. The distribution of phase and amplitude on 60 QD are more regular and some of the variations are observed more clearly (Kumar et al., 2001).

\section{RESULTS AND DISCUSSION}

The study of the anisotropy of galactic cosmic-ray intensity is an essential tool, as it is expected to carry important information about the origin and the propagation mechanism of the galactic cosmic rays. Numerous studies are concentrated on the diurnal variation of cosmic ray intensity (Alania, et al., 2005; Amenomori et al., 2005; Braun et al., 2005; Kozyarivsky etal., 2005; Moraal et al., 2005).

Annual average values of the amplitude of first three harmonics of daily variation in cosmic ray intensity along with statistical error bars on 60 quiet days has been plotted for two different neutron monitoring stations, Deep River with middle cut off rigidity $(1.02 \mathrm{GV})$ and Inuvik with low cutoff rigidity $(0.18 \mathrm{GV})$ in Fig. $1(\mathrm{a}, \mathrm{b}, \mathrm{c})$. One can clearly see from the plots that the amplitude of first harmonic $\left(\mathrm{A}_{1}\right)$ remains high for Deep River as compared to Inuvik neutron monitor having low cutoff rigidity throughout the period of investigation except for the year 1991. The amplitude is found to remain low ( $\sim 0.2 \%$ ) showing dips during the years 1983,1987 and 1991, whereas, remains high $(\sim 0.4 \%)$ showing peaks during 1985 and 1989 at Deep River. The amplitude $\mathrm{A}_{1}$ remains low ( $0.2 \%$ ) showing dips during 1983, 1986 and 1992, whereas, it remains high $(\sim 0.4 \%)$ showing peaks during 1985 and 1992 at Inuvik station. Thus the diurnal amplitude $A_{1}$ significantly decreases during 1986-87 i.e. solar activity minimum years at both Deep River and Inuvik. It is also noticed from the shape of the plots that $A_{1}$ at both the stations is positively correlated during 1981-90, whereas they are anti-correlated from 1990 onwards with each other.

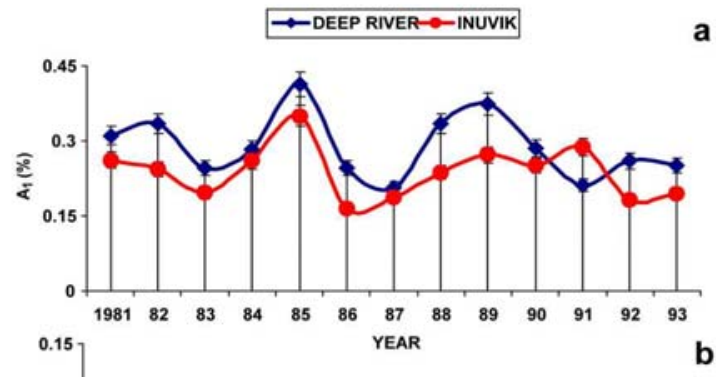

a
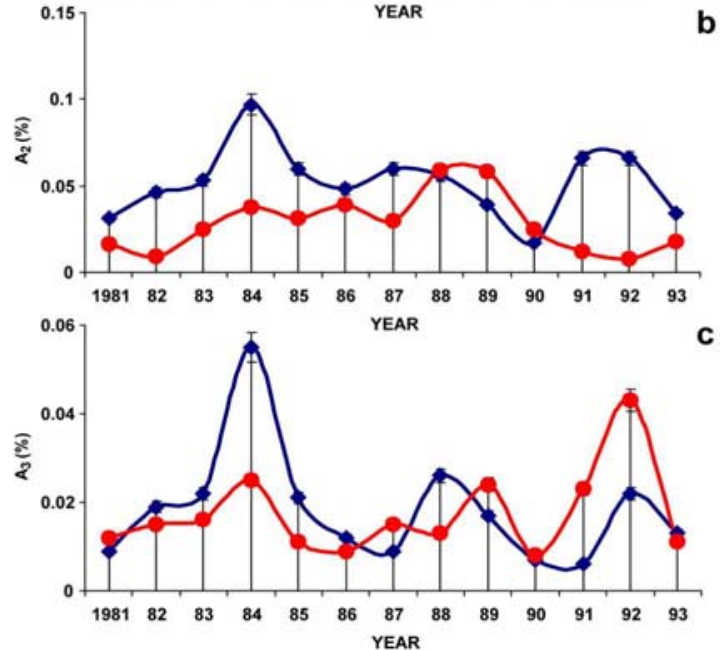

FIG. 1: Average values of the amplitude (\%) of first three harmonics of daily variation in cosmic ray intensity along with statistical error bars (I) on 60 QD for Deep River and Inuvik NM stations.

The semi-diurnal amplitude $\mathrm{A}_{2}$ as depicted in Fig. 1b increases gradually from 1981 and reaches it's maximum $(0.1 \%)$ during 1984 and then decreases up to 1990 at Deep River. 
However $\mathrm{A}_{2}$ increases gradually from 1982 with some fluctuations and reaches it's maximum (0.06\%) during 1988 and then decreases sharply up to 1992 at Inuvik. The semi-diurnal amplitude $A_{2}$ is significantly enhanced during 1984 (solar activity minimum) and 1991-92 (solar activity maximum) at Deep River, whereas $A_{2}$ significantly remains low during 1991-92 (solar activity maximum) at Inuvik. The semidiurnal amplitude $\mathrm{A}_{2}$ is anti-correlated for the two stations during the years 1991-92 as amplitude reaches it's maximum for one station and to minimum for the other during the same year.

The tri-diurnal amplitude $\mathrm{A}_{3}$ as depicted in Fig. 1c increases sharply from 1981 and reaches it's first maximum during 1984 at both the stations having different cutoff rigidity then decreases gradually and second peak occur on 1988 at Deep River and on 1989 at Inuvik. A $_{3}$ decreases sharply from 1988 to 1991 then increases and reaches it's third maximum during 1992, whereas $A_{3}$ start increasing from 1990 and reaches it's third maximum on 1992 at Inuvik. The amplitude $\mathrm{A}_{3}$ significantly enhanced at Deep River during 1984 (solar activity minimum) and at Inuvik during 1992 (solar activity maximum). It is also noticed from the shape of the plots that the amplitude $A_{3}$ is positively correlated with each other for the two stations with different cut off rigidity during the period of investigation.

Annual average values of the time of maximum (Hr) for the first three harmonics of daily variation in cosmic ray intensity on 60 quiet days along with statistical error bars have been plotted for two different neutron monitoring stations Deep River and Inuvik in Fig. 2 (a, b, c). It is clear that the time of maximum (phase) $\phi_{1}$ of diurnal anisotropy shifts towards a later time at Inuvik as compared to the phase at Deep River throughout the period of investigation. $\phi_{1}$ significantly shifts towards an earlier time during 1992 (solar activity maximum) at both the stations. The time of maximum at both the stations seems to be positively correlated with each other during the entire period. To further confirm these trends we also calculated the correlation coefficient between these two phases and we found a significant positive correlation $(r=0.77) . \phi_{1}$ remains along the corotational direction $(18 \mathrm{Hr})$ at Inuvik and shifts towards earlier hours as compared to corotational direction at Deep River for majority of the years.

The time of maximum of semi-diurnal anisotropy $\phi_{2}$ significantly fluctuates during 1981-90 and significantly shifts towards an earlier time during 1991 (solar activity maximum) and then shifts towards later hours from 1992 onwards at Inuvik. The phase $\phi_{2}$ reaches to its maximum $(\sim 06 \mathrm{Hr})$ during 1980 and 1989 and to its minimum $(\sim 02 \mathrm{Hr})$ during 1991 at Deep River station. It is also noteworthy that the time of maximum significantly shifts towards an earlier time at Deep River station as compared to the time of maximum at Inuvik station throughout the period. It is also observed that the time of maximum $\phi_{2}$ changes quite frequently from higher to lower values at Deep River throughout the period. $\phi_{2}$ significantly shift towards an earlier time during 1991 (solar activity maximum) at both the stations with different cut off rigidity. The time of maximum at both the stations seems to be positively correlated with each other during the entire period. To further confirm these trends we also calculated the correlation coeffi-
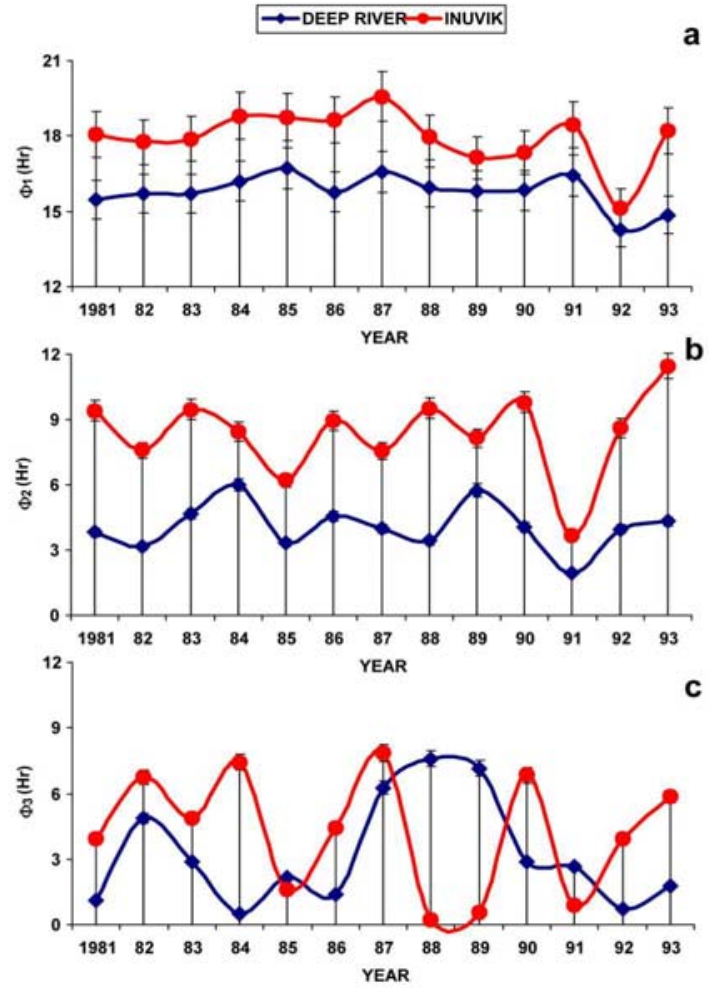

FIG. 2: Average values of the phase $(\mathrm{Hr})$ of first three harmonics of daily variation in cosmic ray intensity along with statistical error bars (I) on 60 QD for Deep River and Inuvik NM stations.

cient between these two phases and we found a good positive correlation $(\mathrm{r}=0.52)$.

The time of maximum $\phi_{3}$ of tri-diurnal anisotropy reaches to its maximum ( $\sim 07 \mathrm{Hr}$ ) on 1982, 1984, 1987, 1990 showing peaks during these years and to its minimum during 1985 , 1988-89, 1991 showing dips during these years at Inuvik station. On the other hand the phase $\phi_{3}$ reaches to its maximum on 1982, 1987-89 showing peaks during these years and to its minimum $(\sim 0.50 \mathrm{Hr})$ on 1980,1984 showing dips during these years. As seen from the figure it is also noteworthy that the time of maximum for these two stations is found to positively correlated during 1981-87 and anti-correlated for the period 1988-1993. To further confirm these trends we have also calculated the correlation coefficient between these two phases and we found a good positive correlation $(r=0.46)$ for 1981-87 and high anti-correlation $(r=-0.69)$ during 1988-93.

Solar wind and interplanetary magnetic field (IMF) plays an important role in controlling the electrodynamics of the heliosphere (Parker, 1964). Solar wind speed, V and IMF parameters, such as vector $\mathbf{B}$, spiral angle and tilt are important for the transport of energetic cosmic ray particles in the heliosphere, for the modulation of cosmic ray and creation of cosmic ray anisotropy in the interplanetary space. The solar wind velocity determines two components of the cosmic ray modulation mechanism: the convection and the adiabatic energy changes. The high velocity solar wind fluxes associated with coronal holes give rise to both isotropic and anisotropic variations in cosmic ray intensity (Iucci et al., 1979; Kaminer 
et al., 1981). Changes of the solar wind velocity near the Earth may have not only local but also the global character (Sheeley et al., 1991; Richardson et al., 1999). Kondoh et al. (1999) found that the peak solar wind velocity has good anticorrelation with the high-energy galactic cosmic ray intensity. The IMF magnitude and fluctuations are responsible for the depression of cosmic ray intensity during high-speed solar wind events (Sabbah, 2000a). The IMF magnitude reaches the highest value during declining phase of solar activity (Sabbah, 1996). The correlation between cosmic ray intensity and solar wind velocity is statistically significant, especially in the period of the maximum solar activity. The regression coefficients obtained on yearly basis depend on sunspot number and are $\sim-0.8$ and $\sim-0.2$ per $100 \mathrm{~km} / \mathrm{s}$ at the solar maximum and minimum, respectively (Fujimoto et al., 1983). The relation of cosmic ray intensity to solar wind velocity is, in general, dependent on physical conditions in the interplanetary space varying with the solar activity. The year-to-year variation of the effect of solar wind upon cosmic ray intensity is dependent on solar activity and the decrement of cosmic ray intensity due to the variation of solar wind velocity is proportional to sunspot number (Fujimoto et al., 1983).

To find out the possible dependence of amplitude and time of maximum on solar wind and IMF, we have plotted the scatter diagram between amplitude/phase and solar wind velocity $(\mathrm{V})$, north south component of IMF (Bz), the product ( $\mathrm{V} \times \mathrm{Bz})$ for the two neutron monitoring stations.

The amplitude (\%) and phase (Hr) of cosmic ray diurnal/semi-diurnal/tri-diurnal anisotropy along with the variation in associated value of solar wind velocity $(\mathrm{V})$ on quiet days and the regression line has been shown in Fig. 3 (a, b, c) for Deep River during 1981-93. The amplitude $\mathrm{A}_{1}$ slightly decreases as the solar wind velocity increases and shows very weak negative correlation $(r=-0.04)$. The phase $\phi_{1}$ significantly remains in a direction earlier than corotational/18-Hr direction and slightly shifts to later time with the increase of solar wind velocity and shows a weak correlation $(r=0.15)$ as depicted in Fig. 3a. The amplitude $A_{2}$ of semi-diurnal anisotropy increases with the increase of solar wind velocity and shows a good positive correlation $(\mathrm{r}=$ 0.34 ). The direction of the semi-diurnal anisotropy $\phi_{2}$ is observed to shift towards earlier hours with the decrease of solar wind velocity and shows some positive correlation $(r=0.20)$ as depicted in the Fig. $3 \mathrm{~b}$. The amplitude $\mathrm{A}_{3}$ of tri-diurnal anisotropy on quiet days is observed to increase with the increase of solar wind velocity and shows positive correlation $(r=0.45)$. The phase $\phi_{3}$ of the tri-diurnal anisotropy shifts towards an earlier time with increase of $\mathrm{V}$ and shows some anti-correlation $(\mathrm{r}=-0.25)$ with $\mathrm{V}$ as depicted in Fig. 3c. Thus, from the above investigations we may infer that only the amplitude of semi/tri-diurnal anisotropy have a good positive correlation, while the others (i.e. amplitude and phase) have very weak correlation with solar wind velocity on quiet days at Deep River station during 1981-1993. It is also observed from these plots that the solar wind velocity significantly remains in the range 350 to $425 \mathrm{~km} / \mathrm{s}$ i.e. being nearly average on quiet days.

The amplitude (\%) and phase (Hr) of cosmic ray diurnal/semi-diurnal/tri-diurnal anisotropy along with the variation in associated value of solar wind velocity (V) and the regression line is plotted and shown in Fig. 4 (a, b, c) for Inuvik on quiet days during 1981-93. As depicted in Fig. 4a the amplitude $A_{1}$ of the diurnal anisotropy is found to slightly increase as the solar wind velocity increases and shows some positive correlation $(r=0.17)$. The phase $\phi_{1}$ is found to remain along the co-rotational/18- $\mathrm{Hr}$ direction and slightly shifts to later time with the increase of solar wind velocity and shows a weak correlation $(r=0.05)$ as depicted in Fig. 4a. The amplitude $A_{2}$ of semi-diurnal anisotropy slightly decreases with the increase of solar wind velocity and shows a weak anti-correlation $(\mathrm{r}=-0.13)$. The direction of the semi-diurnal anisotropy $\phi_{2}$ is observed to shifts towards earlier hours with the increase of solar wind velocity and shows weak anti-correlation $(\mathrm{r}=-0.17)$ as depicted in the Fig. 4b. The amplitude $\mathrm{A}_{3}$ of tri-diurnal anisotropy on quiet days is observed to slightly increase with the increase of solar wind velocity and shows a weak correlation $(r=0.11)$. The phase $\phi_{3}$ of the tri-diurnal anisotropy found to slightly shift towards later hours with the increase of solar wind velocity and shows a weak correlation $(\mathrm{r}=0.09)$ with $\mathrm{V}$ as depicted in Fig. 4c. Thus from the above investigations we may infer that neither the amplitude nor the direction of all the three harmonics have any significant trend associated with solar wind velocity on quiet days at Inuvik station with low cut off rigidity during 1981-1993. It is also observed from these plots that the solar wind velocity significantly remains in the range 350 to 425 $\mathrm{km} / \mathrm{s}$ i.e. being nearly average on quiet days.

Usually, the velocity of high-speed solar wind streams (HSSWSs) is $700 \mathrm{~km} / \mathrm{s}$ (Munakata et al., 1987). Therefore, it may be deduced from these plots that the amplitude as well as direction of first three harmonics on quiet days do not depend on the HSSWS or by the sources on the Sun responsible for producing the HSSWS such as polar coronal holes $(\mathrm{PCH})$ etc. Thus, we may infer that the amplitude and direction of the anisotropy on quiet days are weakly dependent on HSSWSs for two neutron monitoring station of mid and low cutoff rigidity threshold, which is in agreement with earlier findings (Munakata et al., 1987) and significantly contradicts with the earlier results reported by Iucci et al. (1983) and Dorman et al. (1984), that the solar diurnal amplitude is enhanced during the HSSWSs coming from coronal holes. According to Ahluwalia and Riker (1987) there is no relation between solar wind speed and diurnal variation in high rigidity region. The modulation of solar diurnal anisotropy is weakly or less dependent on the solar wind velocity (Munakata et al., 1987).

We have also plotted the scattered diagram (plots not shown here) for the amplitude (\%) and phase (Hr) of cosmic ray diurnal/semi-diurnal/tri-diurnal anisotropy along with the variation in associated value of north south component of IMF $(\mathrm{Bz})$, the product $(\mathrm{V} \times \mathrm{Bz})$ and calculated the correlation coefficient between them on quiet days for Deep River and Inuvik stations. We observed that the semi-diurnal amplitude $\mathrm{A}_{2}$ have a good anti-correlation with $\mathrm{Bz}(\mathrm{r}=-0.40)$ and the product $\mathrm{V} \times \mathrm{Bz}(\mathrm{r}=-0.35)$ at Deep River. The time of maximum of second harmonic $\phi_{2}$ also shows a good anticorrelation with both $\mathrm{Bz}(\mathrm{r}=-0.48)$ and $\mathrm{V} \times \mathrm{Bz}(\mathrm{r}=-0.45)$ 

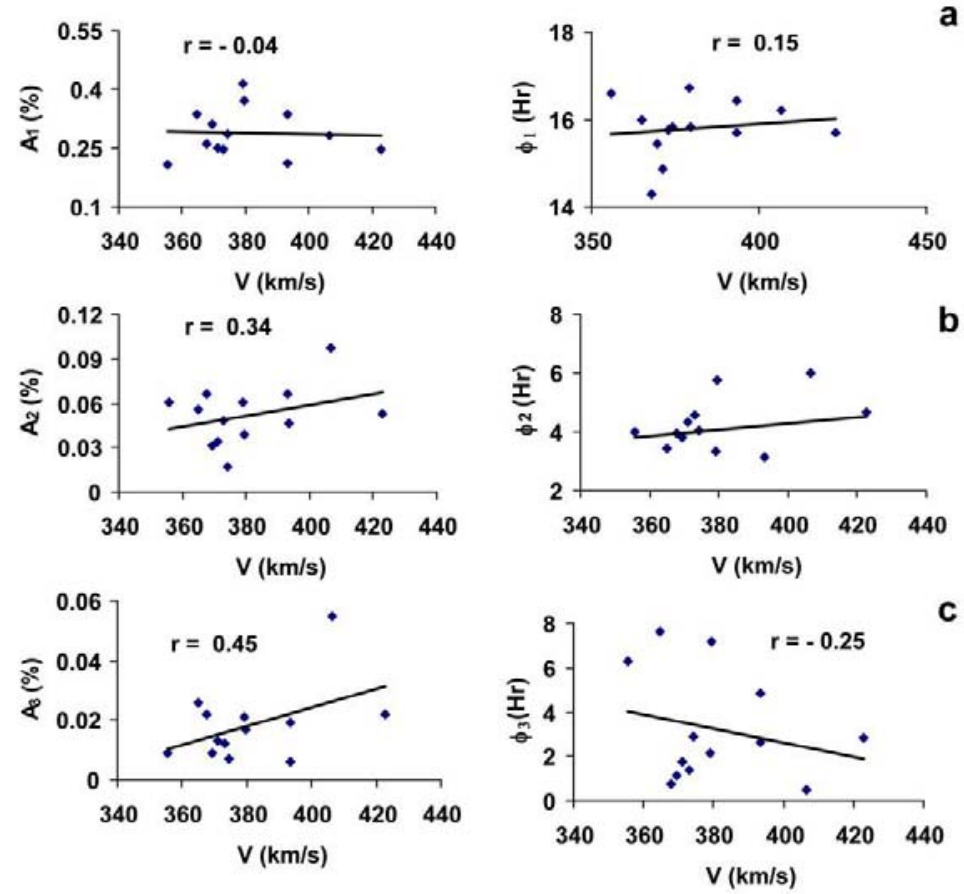

FIG. 3: Amplitude and Phase of the (a) diurnal, (b) semi-diurnal and (c) tri-diurnal anisotropy on quiet days along with solar wind velocity, regression line and correlation coefficient (r) at Deep River station during 1981-1993.

at Deep River. The other components (amplitude and phase) shows very weak correlation with $\mathrm{Bz}$ and $\mathrm{V}$ X Bz.

On the other hand the amplitude of first harmonic $A_{1}$ shows some positive correlation with north south component $\mathrm{Bz}(\mathrm{r}=$ $0.36)$ and the product $\mathrm{V} \times \mathrm{Bz}(\mathrm{r}=0.36)$ at Inuvik. The time of maximum of second and third harmonic $\left(\phi_{2}, \phi_{3}\right)$ shows a good anti-correlation with north south component $\mathrm{Bz}(\mathrm{r}=-0.62$, 0 . 46) and the product $\mathrm{V} \times \mathrm{Bz}(\mathrm{r}=-0.63,-0.44)$ at Inuvik. While the remaining parameters (i.e. amplitude and phase) does not show any significant characteristics associated with $\mathrm{Bz}$ and $\mathrm{V} \mathrm{x} \mathrm{Bz}$ on quiet days. Thus, from the above findings we may infer that the amplitude as well as direction of second harmonic have a good anti-correlation with IMF Bz and the product $\mathrm{V} \times \mathrm{Bz}$ on quiet days at Deep River station. However, the direction of second and third harmonic has a good anticorrelation with IMF Bz and the product $\mathrm{V} x \mathrm{Bz}$ on quiet days at Inuvik station.

Sabbah (1999) obtained an inverse correlation between cosmic ray intensity and the geomagnetic activity and observed the enhancement in upper cut-off rigidity, Rc and the geomagnetic activity resulting from variation in the solar plasma parameters. Upper cut-off rigidity correlates well with the product VB rather than with magnetic field B since VB reflects both diffusion by the IMF and convection with solar wind. The product VB is more important for cosmic rays and geomagnetic activity modulation rather than IMF alone. The ampli- tude of 27-day variation of GCR is also linearly correlated with the IMF strength $\mathrm{B}$, the $\mathrm{z}$-component $\mathrm{Bz}$ of the IMF vector and the product VB (Sabbah, 2000b). Burlaga and Ness (1998) argued that it is ultimately the strong magnetic field and their associated fluctuations that produce the modulation of cosmic rays. Coupling between the IMF strength B and the CR transport parameters leads to a simple modulation model in which the modulation process is linked to global variations of B. Belov (1999) suggested that the local value of the IMF place a significant role in controlling the GCR modulation at an observing site.

\section{CONCLUSIONS}

On the basis of above investigations following important conclusions may be drawn:

The amplitude of first harmonic remains high for Deep River having mid cutoff rigidity as compared to Inuvik neutron monitor having low cutoff rigidity on quiet days. The diurnal amplitude significantly decreases and phase shifts towards an earlier time during solar activity minimum years at both Deep River and Inuvik.

The amplitude of second harmonic significantly enhanced during solar activity minimum as well as maximum at Deep River and remains low during solar activity maximum at In- 

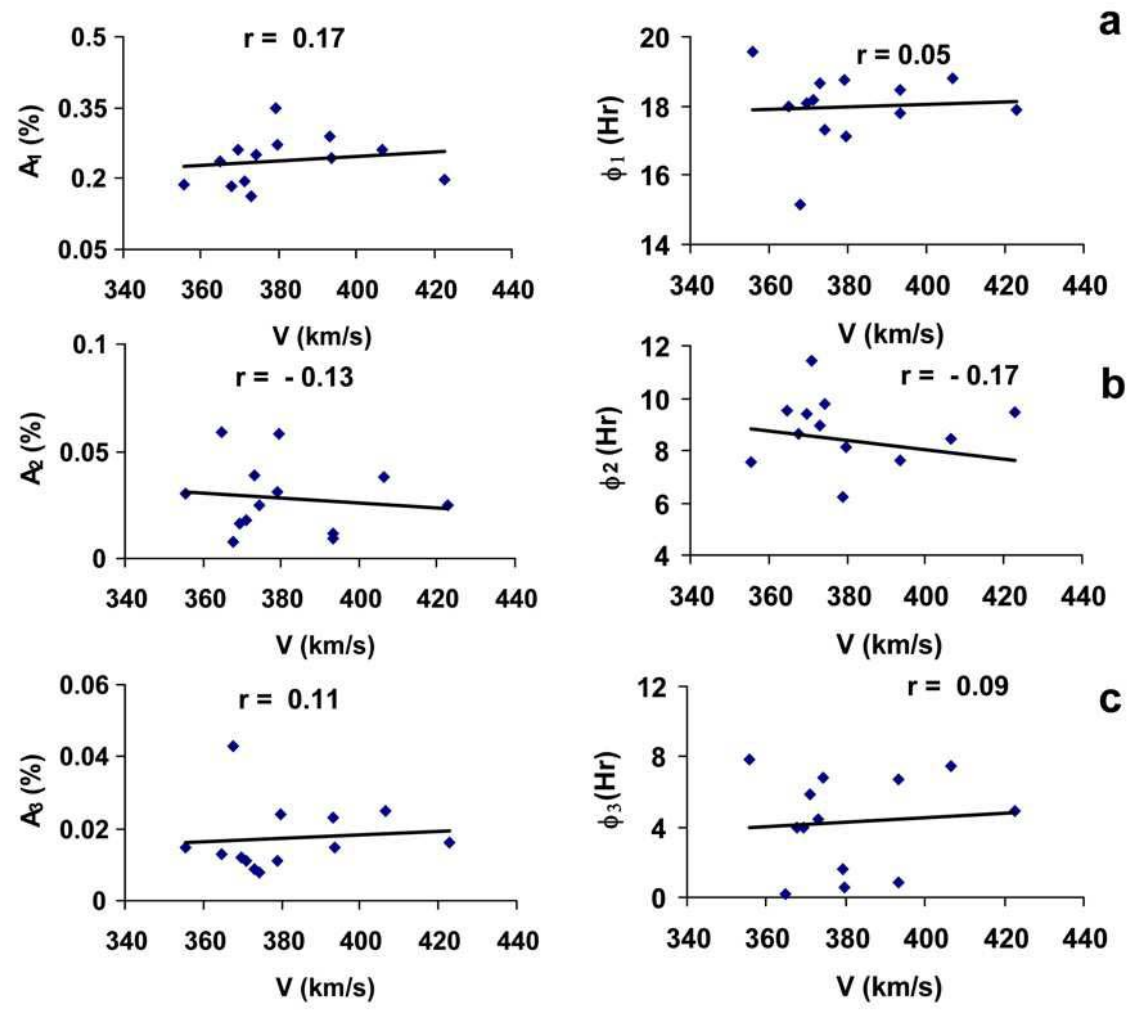

FIG. 4: Amplitude and Phase of the (a) diurnal, (b) semi-diurnal and (c) tri-diurnal anisotropy on quiet days along with solar wind velocity, regression line and correlation coefficient (r) at Inuvik station during 1981-1993.

uvik, whereas the phase shifts towards an earlier time during solar maximum for both the stations.

The amplitude of the third harmonic significantly enhanced during solar activity minimum at Deep River and on solar activity minimum at Inuvik, whereas the phase does not show any significant characteristics and fluctuates quite frequently.

The amplitude of semi/tri-diurnal anisotropy has a good positive correlation, while the others (i.e. amplitude and phase) have very weak correlation with solar wind velocity on quiet days at Deep River.

The amplitude and direction of the anisotropy on quiet days are weakly dependent on high-speed solar wind streams for two neutron monitoring station of mid and low cutoff rigidity threshold.

The amplitude as well as direction of second harmonic has a good anti-correlation with IMF $\mathrm{Bz}$ and the product $\mathrm{V} \times \mathrm{Bz}$ on quiet days at Deep River station. However, the direction of second and third harmonic has a good anti-correlation with IMF $\mathrm{Bz}$ and the product $\mathrm{V} \times \mathrm{Bz}$ on quiet days at Inuvik station.

Additional studies can aid in understanding the results reported here and help to exploit this information to constrain models of solar modulation. Studies of correlations between the CR intensity and IMF/SWP parameter(s) should be useful for identifying the parameter(s) that control the amplitude of the intensity modulation. It will also be of interest to determine how the correlation slopes depend on the time scale over which the data are averaged since the spatial extent of the structures in the heliosphere that control the modulation on short time scales must be smaller than those that produce long term effects.

\section{ACKNOWLEDGEMENTS}

The authors are indebted to various experimental groups, in particular, Prof. Margret D. Wilson, Prof. K. Nagashima, Miss. Aoi Inoue and Prof. J. H. King for providing the data. We also acknowledge the use of NSSDC OMNI database and NGDC geophysical data. The authors are also very much thankful to the anonymous referees for their useful comments/suggestions. 
Agrawal, S.P., Ananth, A.G., Bemalkhedkar, M.M., Kargathra, L.V. and Rao, U.R. 1974. High-energy cosmic ray intensity increase of non-solar origin and the unusual Forbush decrease of August 1972. Journal of Geophysical Research. 79: 2269-2280. Agrawal, S.P., Pathak, S.P., and Mishra, B.L. 1983. $18^{\text {th }}$ International Cosmic Ray Conference. 3: 304-307.

Ahluwalia, H. S. 1988. Is there a twenty year wave in the diurnal anisotropy of cosmic rays. Geophysical Research Letters. 15: 287-290.

Ahluwalia, H. S. and Fikani, M. M., 1997. 25 ${ }^{\text {th }}$ International Cosmic Ray Conference. 2: 125-128.

Ahluwalia, H.S. and Riker, J.F. 1987. Secular changes in the upper cut-off rigidity of the solar diurnal anisotropy. Planetary and Space Science. 35: 39-43.

Alania, M.V., Iskra, K., Modzelewska, R. and Siluszyk, M. 2005. The Galactic Cosmic Ray Intensity and Anisotrophy Variations for Different Ascending and Descending Epochs of Solar Activity. $29^{\text {th }}$ International Cosmic Ray Conference. Edited by B. Sripathi Acharya, Sunil Gupta, P. Jagadeesan, Atul Jain, S. Karthikeyan, Samuel Morris, and Suresh Tonwar. Mumbai: Tata Institute of Fundamental Research. 2: 219-222.

Amenomori, M. et al. 2005. Two dimensional observation on $\mathrm{TeV}$ Cosmic-ray large scale anisotropy using the Tibet Air Shower Array. 29 $9^{\text {th }}$ Interenational Cosmic Ray Conference. Edited by B. Sripathi Acharya, Sunil Gupta, P. Jagadeesan, Atul Jain, S. Karthikeyan, Samuel Morris, and Suresh Tonwar. Mumbai: Tata Institute of Fundamental Research. 2: 49-52.

Axford, W.I. 1965a. The modulation of galactic cosmic rays in the interplanetary medium. Planetary and Space Science. 13: 115 -

Axford, W.I., 1965b. Anisotropic diffusion of solar cosmic rays. Planetary and Space Science. 13: 1301-.

Ballif, J. R., Jones, D. E. and Coleman, P. J. 1969. Further evidence on the correlation between transverse fluctuations in the interplanetary magnetic field and Kp. Journal of Geophysical Research. 74: 2289-2301.

Belov, A.V., Guschina, R.T., Yanke, V.G. 1999. On Connection of Cosmic Ray Long Term Variations with Solar-Heliospheric Parameters. $26^{\text {th }}$ International Cosmic Ray Conference. Edited by D. Kieda, M. Salamon, and B. Dingus. 7: 175-178.

Bieber, J. W. and Evenson, P. 1997. $25^{\text {th }}$ International Cosmic Ray Conference. 2: 81-84.

Braun, J. Engler, H“orandel, J.R. and Milke, J. 2005. Solar modulation of cosmic rays in the energy range from 10 to $20 \mathrm{GeV}$. $29^{\text {th }}$ International Cosmic Ray Conference. 2: 135-138.

Burlaga, L.F. and Ness, N.F. 1998. Magnetic field strength distributions and spectra in the heliosphere and their significance for cosmic ray modulation: Voyager 1, 1980-1994. Journal of Geophysical Research. 103: 29719- 29732.

Bussoletti, E. 1973. Eldo-Celes/Esro-Cers Scient. Technical Review. 5: 285.

Chapmen, S. and Bartels. 1940. Geomagnetic II, Oxford University Press.

Dorman, L. I., Kaminer, N.S., Kuj'micheva, A.E. and Mymrina, N.V. 1984. Features of diurnal variations of cosmic rays in highspeed streams of the solar wind. Geomagnetism and Aeronomy. 24: 546-551.

El-Borie, M. A., Sabbah, I., Darwish, A. and Bishara, A. 1995. $24^{\text {th }}$ International Cosmic Ray Conference. 4: 603-606.

Forbush, Schott E. 1973. Cosmic ray diurnal anisotropy 1937 1972. Journal of Geophysical Research. 78: 7933-7941.
Forman, M.A. and Gleeson, L.J. 1975. Cosmic-ray streaming and anisotropies. Astrophysics and Space Science. 32: 77-94.

Fujimoto, K., Kojimatt, K. and Munakami, K. 1983. Cosmic Ray Intensity Variations and Solar Wind Velocity. $18^{\text {th }}$ International Cosmic Ray Conference. 3: 267-270.

Hashim, A. and Thambyahpillai, H. 1969. Large amplitude wave trains in the cosmic ray intensity. Planetary and Space Science. 17: $1879-1889$.

Iucci, N., Parisi, M., Storini, M. and Villoresi, G. 1983. The behavior of the cosmic-ray equatorial anisotropy inside fast solarwind streams ejected by coronal holes. Nuovo Cimento. 6C: 145148.

Iucci, N., Parisi, M., Storini, M., Villoresi, G. 1979. High-speed solar-wind streams and galactic cosmic-ray modulation. Nuovo Cimento. 2C: 421-438.

Jadhav, D. K., Shrivastava, M., Tiwari, A. K. and Shrivastava, P. K. 1983. Study of semi-diurnal variation of cosmic rays during days of high amplitude wave trains. $18^{\text {th }}$ International Cosmic Ray Conference. 3: 337-340.

Kaminer, N.S., Kuzmicheva, A. E., Mymrina, N.V. 1981, Cosmic-ray anisotropy near the boundary of a high-speed solarwind stream. Geomagnetizm i Aeronomiia. 21: 424-427.

Kane, R. P. 1970. Diurnal anisotropy of cosmic ray intensity. Journal of Geophysical Research. 75: 4350-4353.

Kane, R. P. 1974. Relationship between interplanetary plasma parameters and geomagnetic Dst. Journal of Geophysical Research. 79: 64-72.

Kondoh, K., Hasebe, N., Doke, T., Kikuchi, J., Kobayashi, M. N., Medina J., Sequeiros, J., Takashima, T., Yanagimachi, T., Wilken, B. 1999. Galactic Cosmic Ray and Recurrent Enhancement of Solar Wind Velocity. $26^{\text {th }}$ International Cosmic Ray Conference. Edited by D. Kieda, M. Salamon, and B. Dingus. 7: $179-182$

Kozyarivsky, V.A., Lidvansky, V.A., Petkov, V.B. and Tulupova, T.I. 2005. Mean Diurnal Variations of Cosmic Ray Intensity as Measured by the Baksan Surface and Underground Detectors. $29^{\text {th }}$ International Cosmic Ray Conference. 2: 93-96.

Kumar, S. and Yadav, R.S. 1981. $17^{\text {th }}$ International Cosmic Ray Conference. Edited by B. Sripathi Acharya, Sunil Gupta, P. Jagadeesan, Atul Jain, S. Karthikeyan, Samuel Morris, and Suresh Tonwar. Mumbai: Tata Institute of Fundamental Research. 10: 242-245.

Kumar, S., Agarwal, R., Mishra, R. and Dubey, S.K. 2001. A new concept of analysis of solar daily variation in cosmic ray intensity. $27^{\text {th }}$ International Cosmic Ray Conference. 3: 39663969.

Kumar, S., Chauhan, M. L. and Dubey, S. K. 1997. Effect of Interplanetary Turbulences Causing High/low Amplitude Anisotropic Wave Trains in CR Intensity. Solar Physics. 176: 403-415.

Kumar, S., Gulati, U., Khare, D., Tripathi, M. and Sharma, M. 1993. Study of 22-year periodicity in cosmic ray diurnal anisotropy on quiet days Journal of Pure and Applied Physics. 5: 276-285.

Kumar, S., Shrivastava, S.K., Dubey, S.K., Richharia, M.K. and Gulati, U. 1998. Effect of solar poloidal magnetic field reversal on diurnal anisotropy of cosmic ray intensity on quiet days. Indian Journal of Radio and Space Physics. 27: 236-240.

Lockwood, J. A. and Webber, W. R. 1984. Observations of the dynamics of the cosmic ray modulation. Journal of Geophysical Research. 89: 17-25. 
Mavromichalaki, H. 1979. Astrophysics and Space Science. 80: 59.

McCraken, K.G., and Rao, U.R. 1965. A survey of the diurnal anisotropy. $9^{\text {th }}$ International Cosmic Ray Conference. 1: 213216.

McCraken, K.G., Rao, U.R. and Ness, N.F. 1968. The InterRelationship of Cosmic Ray Anisotropies and the Interplanetary Magnetic Field. Astronomical Journal. 73: 70.

Moraal, H., Caballero-Lopez, R.A., McCracken, K.G. and Humble, J.E. 2005. $29^{\text {th }}$ International Cosmic Ray Conference. 2: 105-108.

Munakata, K., Mori, S., Ryu, J.Y., Agrawal, S.P. and Venkatesan, D. 1987. $20^{\text {th }}$ International Cosmic Ray Conference. 4: 39-42.

Owens, A.J. and Kash, M.M. 1976. Journal of Geophysical Research. 81: 3471-.

Parker, E.N. 1964. Theory of streaming of cosmic rays and the diurnal variation. Planetary and Space Science. 12: 735.

Pomerantz, M.A., Agrawal, S.P. and Potnis, V.R. 1960. Journal of Franklin Institute. 269: 235-.

Rao, U.R. 1972. Solar Modulation of Galactic Cosmic Radiation. Space Science Review. 12: 719-.

Rao, U.R., Ananth, A.G. and Agrawal, S.P. 1972. Characteristics of quiet as well as enhanced diurnal anisotropy of cosmic radiation. Planetary and Space Science. 20: 1799.

Richardson, I. G., Cane, H. V. and Wibberenz, G. 1999. Journal of Geophysical Research. 104: 12549.

Sabbah, I., Darwish, A.A. and Bishara, A.A. 1998. Characteristics of two-way cosmic ray diurnal anisotropy, Solar Physics. 181: 469-477.

Sabbah, I. 1999. Magnetic cycle dependence of the cosmic ray diurnal anisotropy. Solar Physics. 188: 403-417.

Sabbah, I. 2000a. The influence of transient solar-wind events on the cosmic-ray intensity modulation. Canadian Journal of Physics. 78: 293-302.

Sabbah, I. 2000b. The role of interplanetary magnetic field and solar wind in modulating both galactic cosmic rays and geomagnetic activity. Geophysical Research Letters. 27 (13): 18231826.

Sabbah, I., 1996.Journal of Geophysical Research. 101: 2485-. Sheeley, N.R., Swanson, E.T., Wang, T.M. 1991. Journal of Geophysical Research. 96: 861-.

Sikripin, G. V. and Mamrukova, V. P. 1993. Izvestia of Russian Academy of Sciences, Ser. Phys. 57 (N7): 51.

Tiwari, A.K. 1994. Ph.D. thesis, A.P.S. University, Rewa, India. Tiwari, A.K. 1995. $24^{\text {th }}$ International Cosmic Ray Conference. 3: 948-951.

Venkatesan, D. and Badruddin 1990. Space Science Review. 52: 121. 